\title{
Characterization of Aroma Compounds in Cooked Sorghum Using Comprehensive Two-Dimensional Gas Chromatography- Time-of-Flight Mass Spectrometry and Gas Chromatography- Olfactometry-Mass Spectrometry
}

\author{
Shuang Chen ${ }^{1}, * \mathbb{D}$, Li Wang ${ }^{2}$, Derang $\mathrm{Ni}^{2}$, Lin Lin ${ }^{2}$, Heyu Wang ${ }^{2}$ and Yan $\mathrm{Xu}^{1}$ \\ 1 Key Laboratory of Industrial Biotechnology of Ministry of Education, State Key Laboratory of Food \\ Science \& Technology, School of Biotechnology, Jiangnan University, Wuxi 214122, China; \\ yxu@jiangnan.edu.cn \\ 2 Technique Center of Kweichow Moutai Co. Ltd., Guizhou 564500, China; zmx1019@163.com (L.W.); \\ derangni@163.com (D.N.); eileenjn@126.com (L.L.); moutai2@163.com (H.W.) \\ * Correspondence: shuangchen@jiangnan.edu.cn
}

Citation: Chen, S.; Wang, L.; Ni, D.;

Lin, L.; Wang, H.; Xu, Y.

Characterization of Aroma

Compounds in Cooked Sorghum

Using Comprehensive Two-

Dimensional Gas Chromatography-

Time-of-Flight Mass Spectrometry

and Gas Chromatography-

Olfactometry-Mass Spectrometry.

Molecules 2021, 26, 4796. https://

doi.org/10.3390/molecules26164796

Academic Editor: Domenico

Montesano

Received: 30 June 2021

Accepted: 5 August 2021

Published: 7 August 2021

Publisher's Note: MDPI stays neutral with regard to jurisdictional claims in published maps and institutional affiliations.

Copyright: (c) 2021 by the authors. Licensee MDPI, Basel, Switzerland This article is an open access article distributed under the terms and conditions of the Creative Commons Attribution (CC BY) license (https:// creativecommons.org/licenses/by/ $4.0 /)$

\begin{abstract}
Sorghum is the major raw material for the production of Chinese Baijiu (Chinese liquor) and has a great effect on the flavor of Baijiu. Volatiles in cooked glutinous and non-glutinous sorghum samples were extracted using solid-phase microextraction (SPME) and analyzed via comprehensive two-dimensional gas chromatography-time-of-flight mass spectrometry (GC $\times$ GC-TOFMS) and gas chromatography-olfactometry/mass spectrometry (GC-O/MS). A total of 145 volatile compounds and 52 potent odorant compounds were identified from both sorghum types according to the retention index, MS, aroma, and standards. Based on their aroma features, the compounds were grouped into eight general categories, and the intensities of each aroma group were summed. Moreover, most of the compounds detected in the cooked sorghums were also detected in commercial Chinese Baijiu, indicating that the aroma compounds produced during the sorghum cooking process have a direct and significant influence on the final flavor quality of Baijiu.
\end{abstract}

Keywords: cooked sorghum; aroma compounds; SPME; GC×GC-TOFMS; GC-O/MS

\section{Introduction}

Sorghum one of the oldest crops, has been widely grown in the semiarid regions of the world [1]. It is the fifth most important cereal crop in the world and has been widely used in feed and food industries globally. However, sorghum is the most commonly used raw material for the production of Chinese Baijiu (Chinese liquor) [2]. Chinese Baijiu, also known as sorghum spirit, is a traditional Chinese distilled spirit and the most consumed spirit in the world (over 7 billion liters annually) [3]. Baijiu is usually distilled from fermented sorghum, and the demand for sorghum exceeds 4 million tons per year [3]. The production of Baijiu involves a typical repeated-batch fermentation and distillation process [4]. Fermented sorghum is usually mixed with soaked fresh sorghum to undergo solid-state distillation. Alcohol distillation and cooking of the fresh sorghum occur simultaneously. The aroma compounds generated during the cooking process are directly infused into the freshly produced Baijiu [3]. Therefore, the aroma compounds of cooked sorghum can greatly impact the final flavor quality of the Baijiu [5]. Depending on its amylose content, sorghum can be classified as non-glutinous or glutinous [5]. Both types of sorghums are widely used for Baijiu production, with the overall flavor characteristics of the Baijiu produced being significantly different [3].

As a pleasurable alcoholic beverage, the quality of Baijiu is highly dependent on its aroma quality, with the presence of volatile compounds being vital to its aroma characteristics. As far as Baijiu manufacturing is concerned, a significant amount of research on the 
aroma of Baijiu has already been carried out [6,7]. More than 300 aroma-active compounds have been identified in Baijiu, comprising numerous chemical classes, including esters, alcohols, fatty acids, pyrazines, and terpenes [8]. These aroma-active compounds may be derived from the raw materials, fermentation, distillation and aging processes used during production [9]. Since sorghum is the major raw material in the production of Chinese Baijiu, a few authors have identified the volatile compounds of sorghum. Using simultaneous distillation-extraction (SDE) and headspace-solid-phase microextraction (SPME) with gas chromatography-mass spectrometry (GC-MS), Lian et al. [10] identified 108 volatile compounds in sorghum.

However, not all of these compounds are aroma-active compounds. Gas chromatographyolfactometry (GC-O) is the most widely used analytical method for evaluating which volatile compounds are also important odorants in foods. Wu et al. [11] were the first to study the aroma-active compounds of cooked sorghum by GC-O/MS, tentatively identifying a total of 26 aroma-active compounds through comparison with the NIST retention index database. However, the aroma-active compounds that are responsible for the aroma profiles of different types of sorghum have not yet been clearly elucidated.

Therefore, the aims of this work were to: (I) develop an in-vial cooking method to capture and enrich the volatile compounds of cooked sorghum; (II) characterize the overall volatile compound profile by means of GC $\times$ GC-TOFMS; (III) characterize the aroma-active compounds of cooked sorghum by GC-O/MS; and (IV) compare and identify differences in the aroma profiles of glutinous and non-glutinous sorghum. The results of this study could provide a foundation for understanding the aroma role of sorghum as an ingredient in foods and beverages, such as Baijiu.

\section{Results and Discussion}

\subsection{Optimization of the Aroma Extraction Method for Cooked Sorghum}

Solid-phase microextraction (SPME) is widely used for the analysis of aroma compounds due to its relatively simple sample preparation and fully automated operation [12]. In this study, SPME was used to analyze the aroma compounds in cooked sorghum. Before analysis, the SPME conditions were optimized. Firstly, four SPME fibers with different coatings, PDMS, PDMS/DVB, CAR/PDMS, and DVB/CAR/PDMS were evaluated using GC-MS. Among them, the DVB/CAR/PDMS fiber, with a bipolar coating, had the highest extraction yield of aroma compounds. As a result, this fiber was selected to further optimize the extraction temperature and time of the SPME procedure. GC-MS analysis revealed that the volatile compound content of cooked sorghum increases significantly $(p<0.05)$ with increases in the extraction temperature $\left(40-80^{\circ} \mathrm{C}\right)$ (Figure $\left.1 \mathrm{~A}\right)$ and time (30-120 $\left.\mathrm{min}\right)$ (Figure 1B). Therefore, $80^{\circ} \mathrm{C}$ (upper temperature limit of the instrument) and 120 min were determined to be the optimal conditions for aroma extraction.
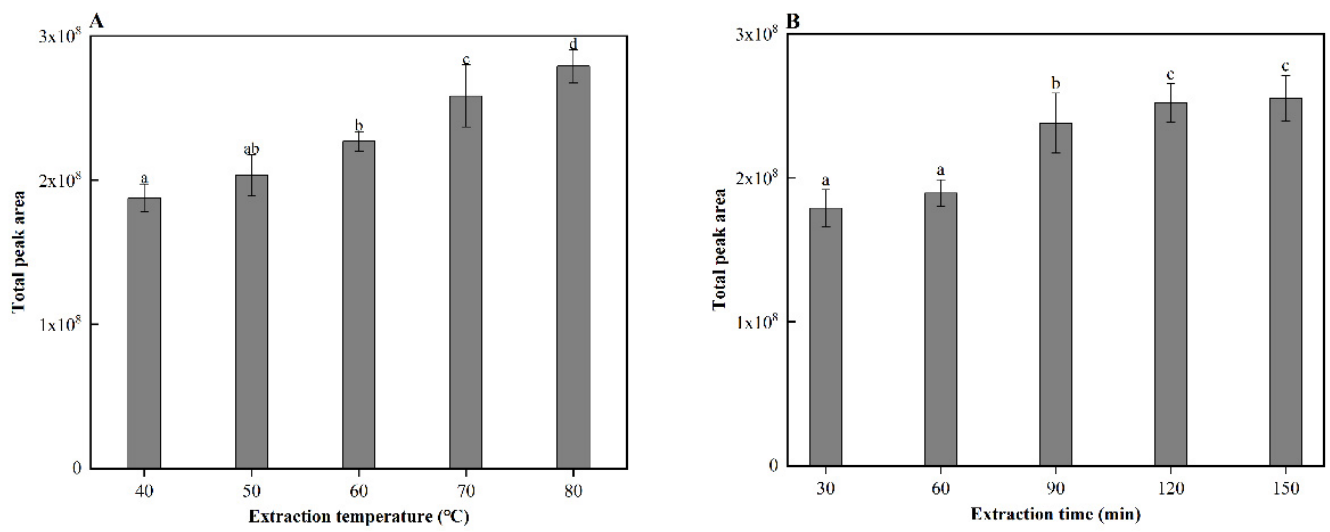

Figure 1. (A) extraction temperatures, and (B) extraction times of solid-phase microextraction (SPME) with corresponding gas chromatography-mass spectrometry (GC-MS) peak areas. Different letters indicate significant differences in values $(p<0.5)$. 


\subsection{Analysis of Volatiles in Cooked Sorghum Samples by Using GC $\times$ GC-TOFMS}

Figure 2 shows the bubble plot obtained by GC $\times$ GC-TOFMS analysis of the volatile components of two cooked sorghum samples. Tentative compound identification was performed by firstly comparing database mass spectra with experimental ones, where database matches were considered with a spectral similarity of higher than $80 \%$, and secondly, comparing between calculated and literature retention values, where a difference value within 50 is desirable. Thirdly, the positive identification of 98 compounds ( $67 \%$ of the total number) was performed with commercially available pure standard compounds injected for qualitative analysis. Finally, a total of 145 volatile compounds were retained in this study, among which aldehydes were present in the highest number (34), followed by alcohol (25), ketones (19), esters (16), terpenes (14), acids (14), heterocyclics (12), sulfides (4), aromatics (3), pyrazines (2), and lactones (2) (Table 1).
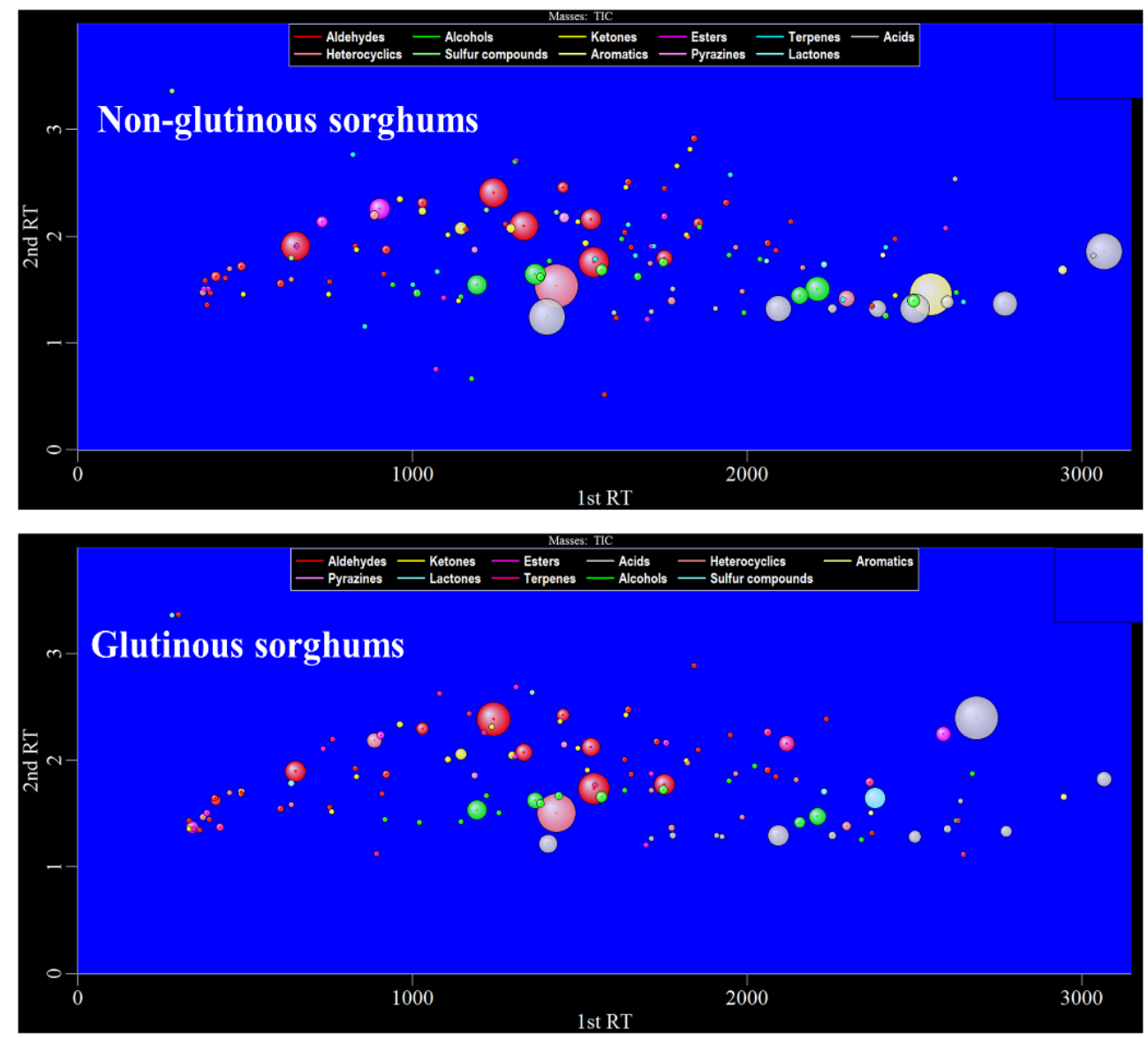

Figure 2. The 2D bubble plot of glutinous and non-glutinous sorghum samples.

As shown in Figure 3, aldehydes were the most abundant class of volatile compounds in the cooked sorghum samples and both saturated and unsaturated linear chain compounds were representative of this class [5]. Aldehydes usually derive from lipid oxidation, and these results could be related to the larger amount of lipids present in the bran layers. Saturated fatty aldehydes which are aromatics, have a greater contribution to the aroma of a cereal product because of their low odor threshold values (OAVs), and provide almond, malt, pungent (pentanal), grassy, green and fatty (hexanal), fatty, citrus and rancid (heptanal), fatty, soapy and green (octanal), fatty, citrus and green (nonanal) characteristics. (E)-2-enal is an important kind of aldehyde, there are $(E)$-2-heptenal, $(E)$-2-octenal, $(E)$-2nonenal and (E)-2-decenal, respectively. Most of them show green, fruity, and fatty odors and the odor characters change with increases of the carbon chain length (Table 2). (E)-2enals were not detected in the free aroma of original sorghum, but were detected in various 
aroma types of Baijiu, indicating that sorghum cooking can promote the introduction of fatty aldehydes into liquor, thus improving the flavor quality of baijiu.

Table 1. Comparison of volatile compounds detected in cooked glutinous and non-glutinous sorghum by HS-SPME-GC $\times$ GC-TOFMS.

\begin{tabular}{cccc}
\hline \multirow{2}{*}{ Class } & \multicolumn{3}{c}{ Number of Compounds } \\
\cline { 2 - 4 } & Glutinous & Non-Glutinous & Total \\
\hline Phenol & 2 & 3 & 3 \\
Pyrazines & 1 & 2 & 2 \\
Lactones & 2 & 2 & 2 \\
Sulfur compounds & 3 & 4 & 4 \\
Acids & 11 & 12 & 14 \\
Heterocyclics & 10 & 12 & 12 \\
Esters & 13 & 8 & 16 \\
Ketones & 12 & 15 & 19 \\
Terpenes & 14 & 14 & 14 \\
Alcohols & 19 & 18 & 25 \\
Aldehydes & 25 & 32 & 34 \\
Total & 112 & 122 & 145 \\
\hline
\end{tabular}

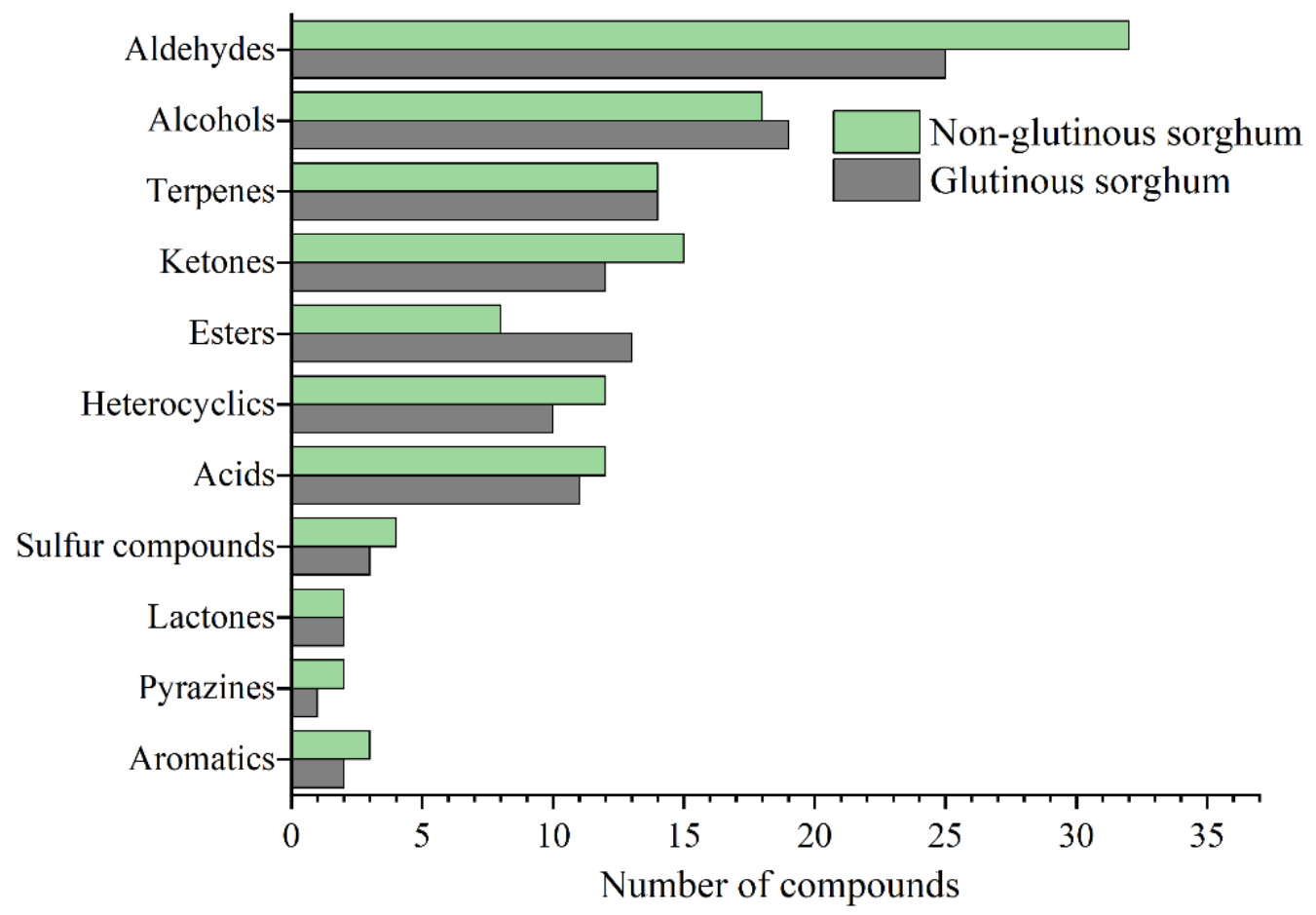

Figure 3. Comparison of identification compounds in cooked glutinous and non-glutinous sorghum obtained by HS-SPMEGC $\times$ GC-TOFMS.

Alcohols are usually produced by the decomposition of the secondary hydroperoxides of fatty acids. Pentanol, hexanol, heptanol and octanol are saturated fatty alcohols, mostly showing grass, flower and fruity odors. 1-octene-3-ol, also known as mushroom alcohol, has a typical mushroom smell, and the threshold in the air is only $1 \mu \mathrm{g} / \mathrm{L}$ [5]. 
Table 2. Volatile compounds identified in cooked glutinous and non-glutinous sorghums by HS-SPME-GC $\times$ GC-TOFMS.

\begin{tabular}{|c|c|c|c|c|c|c|}
\hline \multirow{2}{*}{ Compounds } & \multicolumn{2}{|c|}{ Peak Area } & \multicolumn{2}{|c|}{ RI } & \multirow{2}{*}{ Aroma $^{c}$} & \multirow{2}{*}{ Identification } \\
\hline & Glutinous Sorghum & Non-Glutinous Sorghum & Cla-RI $^{a}$ & Lit-RI $^{b}$ & & \\
\hline \multicolumn{7}{|l|}{ Aldehydes } \\
\hline Acetaldehyde & $9,675,368 \pm 350,920$ & $2,416,216 \pm 104,048$ & 709 & 718 & pungent, ether, fresh & RI, MS, STD \\
\hline Butanal & $903,728 \pm 59,123$ & $22,033,094 \pm 215,879$ & 875 & 854 & pungent, green & RI, MS, Tent \\
\hline 2-Propenal & $95,002 \pm 8637$ & $164,626 \pm 10,770$ & 832 & 838 & almond, cherry & RI, MS, Tent \\
\hline 2-Methyl-2-propenal & $53,945 \pm 4904$ & $95,014 \pm 6216$ & 887 & 893 & wild, hyacinth, foliage & RI, MS, STD \\
\hline 2-Methyl-butanal & $989,169 \pm 64,712$ & $34,559,663 \pm 61,488$ & 904 & 910 & malt & RI, MS, STD \\
\hline 3-Methyl-butanal & $2,194,192 \pm 79,582$ & $2,973,227 \pm 45,278$ & 917 & 916 & cocoa, almond & RI, MS, STD \\
\hline Pentanal & $923,054 \pm 60,387$ & $100,296,059 \pm 69,876$ & 985 & 976 & almond, malt, pungent & RI, MS, Tent \\
\hline 2-Butenal & $162,609 \pm 6776$ & $842,320 \pm 113,039$ & 1056 & 1042 & floral & RI, MS, Tent \\
\hline Hexanal & $2,355,706 \pm 85,440$ & $6,868,006 \pm 104,589$ & 1095 & 1083 & grass, tallow, fatty & RI, MS, STD \\
\hline (E)-2-Pentenal & - & $804,648 \pm 655,501$ & 1125 & 1147 & strawberry, fruity & RI, MS, STD \\
\hline Heptanal & $105,864 \pm 4411$ & $475,576 \pm 63,822$ & 1189 & 1178 & fatty, citrus, rancid & RI, MS, Tent \\
\hline 3-Methyl-2-butenal & $89,259 \pm 8115$ & $101,051 \pm 6611$ & 1212 & 1215 & sweet fruity & RI, MS, Tent \\
\hline 2-Hexenal & $197,246 \pm 8219$ & $1,106,558 \pm 14,849$ & 1207 & 1216 & apple, green & RI, MS, STD \\
\hline Octanal & $1,390,299 \pm 50,426$ & $2,519,793 \pm 38,373$ & 1310 & 1319 & fatty, soapy, green & RI, MS, STD \\
\hline (E)-2-Heptenal & $590,306 \pm 38,618$ & - & 1333 & 1320 & soapy, fatty, almond & RI, MS, STD \\
\hline 2-Octenal & - & $14,342,203 \pm 116,837$ & 1443 & 1445 & fatty green herbal & RI, MS, Tent \\
\hline Decanal & $1,631,776 \pm 59,184$ & $2,340,543 \pm 35,643$ & 1506 & 1506 & soapy, orange peel & RI, MS, STD \\
\hline Nonenal & - & $11,040,264 \pm 89,938$ & 1440 & 1453 & green cucumber & RI, MS, STD \\
\hline (E)-2-Nonenal & $829,816 \pm 54,287$ & - & 1520 & 1535 & cucumber, fatty, green & RI, MS, STD \\
\hline Benzaldehyde & $3,571,972 \pm 129,554$ & $15,600,161 \pm 23,756$ & 1549 & 1524 & almond, burnt, sugar & RI, MS, STD \\
\hline$(E, Z)-2,6-N o n a d i e n a l$ & - & $1,781,208 \pm 14,510$ & 1597 & 1591 & cucumber, wax, green & RI, MS, STD \\
\hline Undecanal & - & $2,441,022 \pm 19,885$ & 1611 & 1609 & oil, pungent, sweet & RI, MS, STD \\
\hline 2,4-Octadienal & - & $832,551 \pm 678,232$ & 1575 & 1585 & green, cucumber & RI, MS, STD \\
\hline (E)-2-Decenal & - & $1,776,196 \pm 14,469$ & 1650 & 1641 & tallow, waxy, fatty & RI, MS, STD \\
\hline Phenylacetaldehyde & $16,153,454 \pm 585,877$ & $26,877,978 \pm 11,574$ & 1658 & 1650 & honey & RI, MS, STD \\
\hline Dodecanal & - & $1,731,008 \pm 141,015$ & 1715 & 1709 & soapy, citrus, green & RI, MS, Tent \\
\hline$(E, E)$-2,4-Nonadienal & $729,557 \pm 47,728$ & $48,460,352 \pm 474,815$ & 1713 & 1686 & fatty, tropical fruity & RI, MS, STD \\
\hline (E)-2-Undecenal & $510,997 \pm 3430$ & $5,568,899 \pm 56,396$ & 1719 & 1736 & soapy, fatty, green & RI, MS, STD \\
\hline$(E, E)$-2,4-Decadienal & $466,460 \pm 30,516$ & $40,596,386 \pm 397,762$ & 1778 & 1783 & fried, wax, fatty & RI, MS, STD \\
\hline 2,4-Dimethyl-benzaldehyde & $96,234 \pm 4010$ & $158,918 \pm 21,327$ & 1748 & 1710 & cherry, almond, vanilla & RI, MS, Tent \\
\hline 2-Methyl-undecanal & $384,132 \pm 3806$ & $357,289 \pm 18,627$ & 1609 & 1636 & waxy, fatty, metallic & RI, MS, Tent \\
\hline 1H-Pyrrole-2-carboxaldehyde & - & $179,875 \pm 46,534$ & 2046 & 2059 & musty, beefy, coffee & RI, MS, Tent \\
\hline
\end{tabular}


Table 2. Cont

\begin{tabular}{|c|c|c|c|c|c|c|}
\hline \multirow{2}{*}{ Compounds } & \multicolumn{2}{|c|}{ Peak Area } & \multicolumn{2}{|c|}{ RI } & \multirow{2}{*}{ Aroma $^{c}$} & \multirow{2}{*}{ Identification } \\
\hline & Glutinous Sorghum & Non-Glutinous Sorghum & Cla-RI $^{a}$ & Lit-RI $^{b}$ & & \\
\hline \multicolumn{7}{|l|}{ Alcohols } \\
\hline 2-Propanol & - & $2,823,662 \pm 23,002$ & 921 & 950 & musty, woody & RI, MS, Tent \\
\hline 2-Methyl-butanol & - & $284,448 \pm 12,249$ & 1200 & 1214 & wine, onion & RI, MS, STD \\
\hline Pentanol & $4,122,806 \pm 149,532$ & - & 1261 & 1275 & fruity & RI, MS, STD \\
\hline 3-methyl-3-butenol & $87,123 \pm 3630$ & $111,599 \pm 14,977$ & 1136 & 1118 & sweet fruity & RI, MS, STD \\
\hline 2-Heptanol & $4,272,658 \pm 54,967$ & - & 1316 & 1280 & mushroom & RI, MS, STD \\
\hline (Z)-2-Pentenol & $99,697 \pm 4154$ & $115,061 \pm 15,441$ & 1310 & 1327 & green, metallic & RI, MS, STD \\
\hline Hexanol & $16,714,881 \pm 60,239$ & $5,390,684 \pm 22,135$ & 1356 & 1350 & resin, flower, green & RI, MS, STD \\
\hline 3-Hexenol & - & $621,386 \pm 506,208$ & 1393 & 1398 & green leafy & RI, MS, Tent \\
\hline 1-Octen-3-ol & $8,675,498 \pm 314,656$ & $6,647,311 \pm 26,248$ & 1442 & 1456 & mushroom & RI, MS, STD \\
\hline Heptanol & $1,929,513 \pm 69,983$ & $431,169 \pm 6566$ & 1440 & 1421 & green & RI, MS, STD \\
\hline 2-Ethyl-hexanol & - & $983,534 \pm 42,353$ & 1490 & 1481 & rose, green & RI, MS, Tent \\
\hline (E)-2-Heptenol & $133,566 \pm 5565$ & - & 1531 & 1546 & pungent, fatty & RI, MS, Tent \\
\hline 2-Nonanol & $980,889 \pm 64,171$ & $8,535,137 \pm 862,710$ & 1555 & 1532 & cucumber & RI, MS, Tent \\
\hline Octanol & $3,985,051 \pm 144,536$ & $1,399,177 \pm 21,308$ & 1565 & 1572 & moss, nut, mushroom & RI, MS, STD \\
\hline (Z)-3-Octenol & $45,855 \pm 4169$ & - & 1566 & 1563 & melon, earthy & RI, MS, Tent \\
\hline 4-Methyl-pentanol & $75,541 \pm 6868$ & - & 1334 & 1316 & nutty & RI, MS, Tent \\
\hline Nonanol & $1,639,336 \pm 59,458$ & $728,439 \pm 11,093$ & 1663 & 1651 & fatty, green & RI, MS, STD \\
\hline Decanol & $194,979 \pm 8124$ & $267,183 \pm 35,856$ & 1761 & 1784 & fatty & RI, MS, Tent \\
\hline 2-Undecanol & $153,446 \pm 6394$ & $291,288 \pm 39,091$ & 1734 & 1717 & fresh wax & RI, MS, Tent \\
\hline 1-Hepten-3-ol & - & $299,299 \pm 43,822$ & 1346 & 1331 & sweet, green & RI, MS, STD \\
\hline Benzenemethanol & $544,118 \pm 35,597$ & $102,365,753 \pm 297,758$ & 1885 & 1902 & sweet, flower & RI, MS, STD \\
\hline Phenylethyl alcohol & $4,612,402 \pm 167,289$ & $6,865,240 \pm 295,633$ & 1900 & 1931 & honey, spice, rose & RI, MS, STD \\
\hline 2-Phenoxy-ethanol & $48,375 \pm 4398$ & - & 2126 & 2142 & rose, cinnamyl & RI, MS, Tent \\
\hline \multicolumn{7}{|l|}{ Ketones } \\
\hline 2-Propanone & - & $493,389 \pm 1936$ & 791 & 816 & solvent, pear & RI, MS, Tent \\
\hline 2-Pentanone & - & $2,038,749 \pm 16,608$ & 963 & 1003 & ether, fruity & RI, MS, Tent \\
\hline 2,3-Butanedione & $940,134 \pm 34,098$ & - & 965 & 977 & butter, sweet, creamy & RI, MS, STD \\
\hline 2,3-Pentanedione & $99,279 \pm 9026$ & - & 1086 & 1062 & sweet, butter, creamy & RI, MS, Tent \\
\hline 2-Heptanone & - & $6,160,141 \pm 18,317$ & 1213 & 1191 & soap & RI, MS, Tent \\
\hline 3-Octanone & - & $4,162,680 \pm 91,099$ & 1226 & 1252 & soap, gasoline & RI, MS, Tent \\
\hline 2-Octanone & $2,021,178 \pm 73,307$ & $4,559,765 \pm 69,438$ & 1261 & 1309 & herb, butter, milk & RI, MS, STD \\
\hline
\end{tabular}


Table 2. Cont

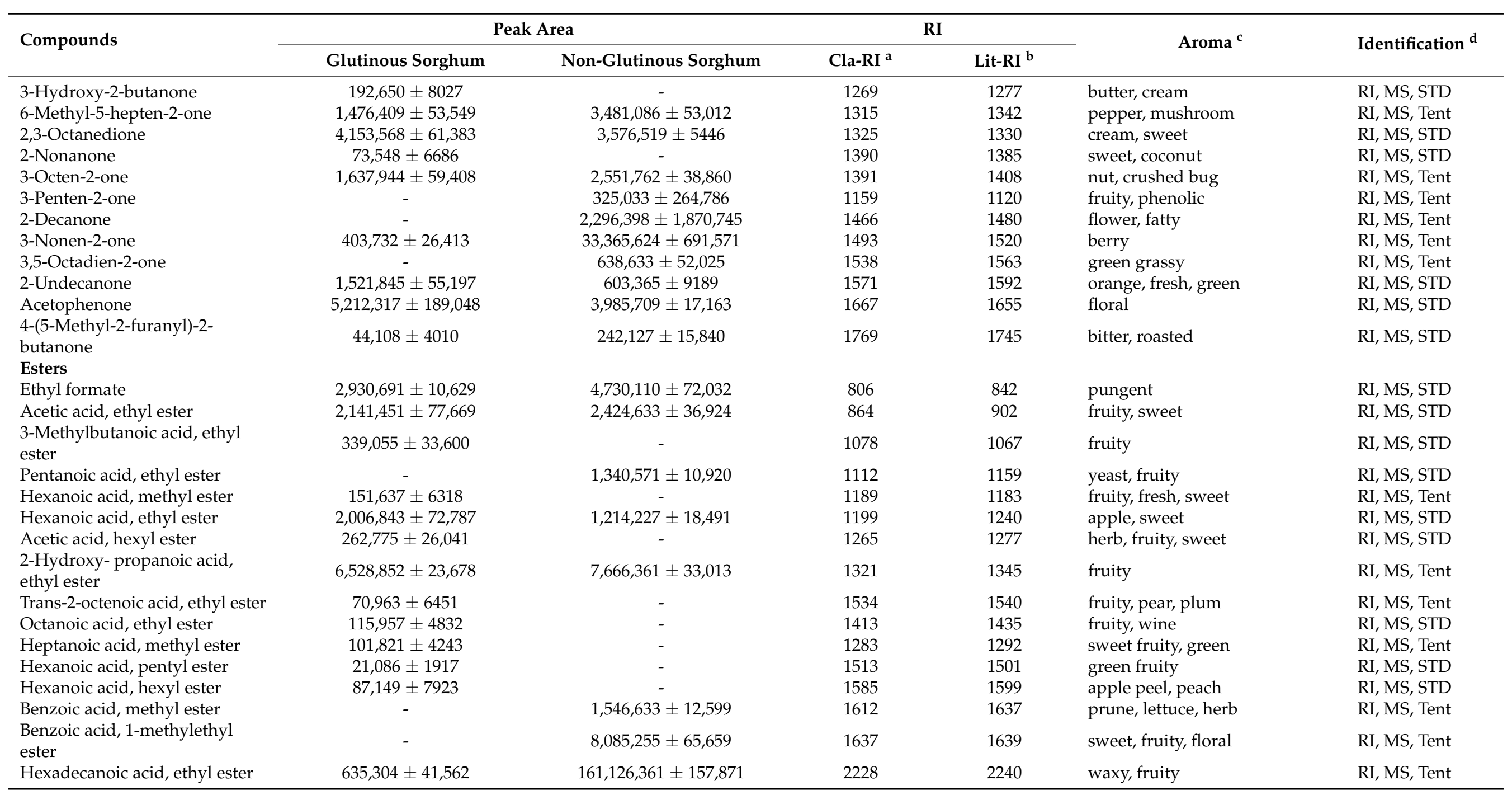


Table 2. Cont

\begin{tabular}{|c|c|c|c|c|c|c|}
\hline \multirow{2}{*}{ Compounds } & \multicolumn{2}{|c|}{ Peak Area } & \multicolumn{2}{|c|}{ RI } & \multirow{2}{*}{ Aroma $^{c}$} & \multirow{2}{*}{ Identification } \\
\hline & Glutinous Sorghum & Non-Glutinous Sorghum & Cla-RI $^{a}$ & Lit-RI $^{b}$ & & \\
\hline \multicolumn{7}{|l|}{ Terpenes } \\
\hline$\alpha$-Terpinene & $383,922 \pm 38,047$ & $276,282 \pm 14,404$ & 1162 & 1172 & citrus-like, floral & RI, MS, STD \\
\hline$\alpha$-Limonene & $273,432 \pm 27,097$ & $161,047 \pm 8396$ & 1193 & 1200 & citrus-like & RI, MS, STD \\
\hline$\gamma$-Terpinene & $116,456 \pm 4853$ & $172,494 \pm 2314$ & 1230 & 1238 & citrus-like, lemon & RI, MS, STD \\
\hline Terpinolene & $154,506 \pm 6438$ & $240,321 \pm 3225$ & 1306 & 1280 & citrus-like, floral & RI, MS, STD \\
\hline$\beta$-Elemene & $168,909 \pm 7038$ & $343,075 \pm 46,041$ & 1598 & 1586 & fruity, sweet & RI, MS, STD \\
\hline$(-)$ - $\alpha$-Cedrene & $180,884 \pm 7537$ & $367,397 \pm 49,305$ & 1568 & 1571 & cypress & RI, MS, STD \\
\hline Thujopsene & $255,330 \pm 25,303$ & $150,385 \pm 7840$ & 1600 & 1606 & cypress & RI, MS, STD \\
\hline$\beta$-Terpineol & $108,746 \pm 4531$ & $161,074 \pm 21,616$ & 1632 & 1616 & clove & RI, MS, STD \\
\hline Linalool & $80,301 \pm 7300$ & $130,720 \pm 8552$ & 1552 & 1535 & flower, wood & RI, MS, STD \\
\hline$\beta$-Damascenone & $402,071 \pm 3984$ & $366,180 \pm 19,090$ & 1832 & 1827 & sweet, honey & RI, MS, STD \\
\hline Geranyl acetone & $578,093 \pm 37,819$ & $14,052,357 \pm 37,684$ & 1861 & 1862 & fruity & RI, MS, STD \\
\hline$(E)$ - $\beta$-ionone & $334,172 \pm 33,116$ & $267,967 \pm 13,970$ & 1952 & 1953 & violet & RI, MS, STD \\
\hline D-nerolidol & $343,040 \pm 33,995$ & $231,477 \pm 12,068$ & 2272 & 2290 & mild floral & RI, MS, STD \\
\hline (E)-Nerolidol & $299,822 \pm 29,712$ & $387,590 \pm 20,206$ & 2040 & 2054 & rose, apple & RI, MS, STD \\
\hline \multicolumn{7}{|l|}{ Acids } \\
\hline Butanoic acid & $805,495 \pm 8566$ & $507,462 \pm 6012$ & 1625 & 1628 & acid, sweaty & RI, MS, STD \\
\hline 3-Methyl-butanoic acid & - & $593,181 \pm 25,544$ & 1660 & 1648 & sweat, acid, rancid & RI, MS, STD \\
\hline Pentanoic acid & $656,331 \pm 42,938$ & - & 1737 & 1733 & acidic, cheesy & RI, MS, STD \\
\hline Hexanoic acid & $32,242,500 \pm 1,169,417$ & $15,491,525 \pm 667,099$ & 1850 & 1857 & fatty, sweat, cheese & RI, MS, STD \\
\hline Heptanoic acid & $8,631,813 \pm 313,071$ & $1,850,130 \pm 79,671$ & 2134 & 2142 & rancid, sour, cheesy & RI, MS, STD \\
\hline Octanoic acid & $11,893,186 \pm 431,359$ & $3,281,620 \pm 141,314$ & 1472 & 1462 & sweat, cheese & RI, MS, STD \\
\hline Nonanoic acid & $11,290,020 \pm 409,483$ & $3,026,817 \pm 130,342$ & 2164 & 2180 & green, fatty & RI, MS, STD \\
\hline Decanoic acid & $1,549,490 \pm 56,199$ & $1,115,042 \pm 16,981$ & 2253 & 2264 & rancid, fatty & RI, MS, STD \\
\hline Hydrocinnamic acid & - & $27,120 \pm 1168$ & 2603 & 2633 & sweet, fatty, rose & RI, MS, STD \\
\hline Hexadecanoic acid & - & $38,378,443 \pm 165,266$ & 2886 & 2876 & waxy, creamy fatty & RI, MS, Tent \\
\hline Benzoic acid & $1,480,735 \pm 53,706$ & $3,555,504 \pm 54,145$ & 2410 & 2404 & urine & RI, MS, STD \\
\hline Tetradecanoic acid & $2,554,084 \pm 92,635$ & $4,176,313 \pm 63,599$ & 2716 & 2734 & fatty, soapy, coconut & RI, MS, Tent \\
\hline \multicolumn{7}{|l|}{ Heterocyclics } \\
\hline 2-Methylfuran & $286,945 \pm 28,436$ & $1,597,938 \pm 83,305$ & 848 & 875 & chocolate & RI, MS, Tent \\
\hline 2,5-Dimethyl-furan & $195,451 \pm 8144$ & $462,775 \pm 62,104$ & 934 & 958 & chemical, meaty, gravy & RI, MS, STD \\
\hline
\end{tabular}


Table 2. Cont

\begin{tabular}{|c|c|c|c|c|c|c|}
\hline \multirow{2}{*}{ Compounds } & \multicolumn{2}{|c|}{ Peak Area } & \multicolumn{2}{|c|}{ RI } & \multirow{2}{*}{ Aroma $^{c}$} & \multirow{2}{*}{ Identification $^{\mathrm{d}}$} \\
\hline & Glutinous Sorghum & Non-Glutinous Sorghum & $\mathrm{Cla}^{-R I^{a}}$ & Lit-RI $^{\mathbf{b}}$ & & \\
\hline 2-Vinylfuran & - & $3,956,245 \pm 32,229$ & 1059 & 1085 & coffee & RI, MS, STD \\
\hline 2-Pentylfuran & $30,803,201 \pm 11,172$ & $17,918,860 \pm 771,626$ & 1209 & 1241 & green bean, butter & RI, MS, STD \\
\hline Furfural & $1,337,092 \pm 48,496$ & $16,802,768 \pm 25,588$ & 1459 & 1486 & almond & RI, MS, STD \\
\hline 2-Acetyl-5-methylfuran & $88,602 \pm 8055$ & $402,960 \pm 26,362$ & 1612 & 1608 & musty, nutty, coconut & RI, MS, STD \\
\hline 2-Furanmethanol & $96,955 \pm 4040$ & $419,643 \pm 56,316$ & 1645 & 1666 & burnt & RI, MS, STD \\
\hline $\begin{array}{l}\text { 1-(5-Methyl-2-furanyl)- } \\
\text { propanone }\end{array}$ & - & $1,748,396 \pm 14,243$ & 1671 & 1686 & green, hazelnut-like & RI, MS, Tent \\
\hline 2-Pentanoylfuran & $176,476 \pm 7353$ & $106,465 \pm 14,288$ & 1750 & 1747 & sweet caramel & RI, MS, Tent \\
\hline $2(5 \mathrm{H})$-Furanone & $106,282 \pm 4429$ & $234,854 \pm 31,517$ & 1761 & 1787 & buttery & RI, MS, STD \\
\hline 2-Hexanoyl furan & $312,315 \pm 30,950$ & $183,949 \pm 9590$ & 1858 & 1872 & sweet, fruity, green & RI, MS, Tent \\
\hline 2-Acetylpyrrole & $1,648,279 \pm 59,782$ & $137,960 \pm 2101$ & 1987 & 2002 & popcorn-like & RI, MS, STD \\
\hline \multicolumn{7}{|l|}{ Sulfur compounds } \\
\hline Methanethiol & $283,822 \pm 28,127$ & $289,490 \pm 15,092$ & 602 & 643 & sulfur, gasoline, garlic & RI, MS, STD \\
\hline Dimethyl disulfide & $316,357 \pm 31,351$ & $891,894 \pm 46,497$ & 1059 & 1078 & onion, cabbage, putrid & RI, MS, STD \\
\hline Dimethyl trisulfide & $338,026 \pm 33,498$ & $763,285 \pm 39,792$ & 1353 & 1400 & sulfur, fish, cabbage & RI, MS, STD \\
\hline 2-Pentyl-thiophene & - & $667,400 \pm 54,360$ & 1422 & 1460 & sweet, fruity & RI, MS, Tent \\
\hline 2-Methoxy-4-vinylphenol & $7,974,386 \pm 28,922$ & $647,661 \pm 27,890$ & 2214 & 2213 & smoky, clove & RI, MS, STD \\
\hline Vanillin & $127,696 \pm 5321$ & $736,429 \pm 98,828$ & 2551 & 2530 & sweet & RI, MS, STD \\
\hline \multicolumn{7}{|l|}{ Pyrazines } \\
\hline 2,6-Dimethyl-pyrazine & - & $4,684,846 \pm 38,164$ & 1335 & 1308 & roasted nut, cocoa & RI, MS, STD \\
\hline Tetramethyl-pyrazine & $59,882 \pm 5444$ & $1,142,923 \pm 74,771$ & 1472 & 1462 & nutty, coffee, burnt & RI, MS, STD \\
\hline \multicolumn{7}{|l|}{ Lactones } \\
\hline$\gamma$-Octalactone & $3,815,443 \pm 13,838$ & $284,300 \pm 4330$ & 1941 & 1924 & coconut milk & RI, MS, STD \\
\hline$\gamma$-Nonalactone & $7,974,386 \pm 28,922$ & $647,661 \pm 27,890$ & 2053 & 2044 & coconut milk & RI, MS, STD \\
\hline
\end{tabular}

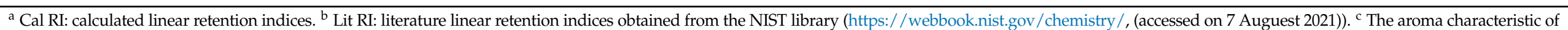
the compound itself. ${ }^{\mathrm{d}}$ Identification: tentative identification (Tent.) based on retention indices (RI) and mass spectra (MS), positive identification based on retention times of authentic standards (STD). 
Ketone compounds formed by the autoxidation of fatty acids were additional major volatile components in the cooked sorghum. Among the various ketones identified in the current study, most of them present butter, cream and fruity odors.

In total, 16 esters were identified in cooked sorghum, and they usually contributed to desirable odors, like fruity, floral and sweety.

Terpenes are a kind of natural hydrocarbon that are widely found in plants. In the current study a total of 14 terpenoid compounds were detected, including mono- and poly-terpene hydrocarbons, alcohols, carbonyls and esters.

A total of 14 acid compounds were identified in this study, of these identified acids, the homologous series of straight-chain monocarboxylic fatty acids from C4-C10, C14, and C16 were all detected, which contributed to acidic, rancid and cheesy odors.

Some heterocycles were also detected in the samples, including furan, furanone and pyrrole. 2-Pentylfuran exists in the cooking process of sorghum and it is derived from lipid peroxidation. This compound has a typical butter odor at a dilute concentration, but at higher concentrations, it has the less pleasant aroma characteristic of soybeans [13]. Furfural (sweet, woody and almond) which has been identified as an aroma-active compound in some cereals, was only detected in cooked samples.

\subsection{Analysis of Aroma-Active Volatiles in Cooked Sorghums by Using GC-O/MS}

The volatile compounds of the cooked glutinous and non-glutinous sorghum samples were directly extracted using the optimized headspace-SPME method and analyzed by GC-O/MS. The analysis results showed that 51 and 48 aroma-active volatiles were detected in glutinous and non-glutinous sorghum, respectively (Table 3). Most of the aroma compounds detected in the cooked sorghums have also previously been identified in Chinese Baijiu $[6,7,14,15]$, indicating that the aroma compounds in sorghum contribute to the overall flavor of Baijiu. The 52 different identified aroma volatiles included 14 aldehydes, 13 terpenes, 8 acids, 5 ketones, 5 alcohols, 5 heterocyclic compounds, 1 sulfurcontaining and 1 aromatic compound. Numerically, the aldehyde group comprised $27 \%$ of the total aroma-active volatiles in the cooked sorghum samples, followed by terpene $(25 \%)$, acid $(14 \%)$, ketone $(10 \%)$, alcohol $(10 \%)$, heterocyclic $(10 \%)$, aromatic $(2 \%)$, and sulphur-containing ( $2 \%)$ compounds. The aroma intensity of $(E)$-2-heptenal, nonanal, $(E)-2-$ octenal, benzaldehyde, $(E)$-2-undecenal, $(E, E)$-2,4-decadienal, $\alpha$-limonene, $(-)-\alpha$-Cedrene, thujopsene, $\beta$-elemene, $\beta$-damascenone, geranyl acetone, 2,3-octanedione, acetophenone, 2-pentylfuran, 2-acetylpyrrole and dimethyl disulfide, present significant differences between the two samples $(p<0.05)$. $(E)$-2-undecenal was undetected in glutinous sorghum, and $(E, E)$-2,4-decadienal, $\alpha$-limonene, thujopsene, and $\beta$-elemene were undetected in non-glutinous sorghum (Figure 4).

To estimate the contributions that these aroma-active volatiles had on the overall aroma impression, the 52 compounds were grouped into eight general aroma categories: sweet/fruity, green, floral, fatty, volatile acids, citrus-like, roasty/nutty, and miscellaneous (sulphury/smoky/mushroom). To compare the differences in the aroma intensities of each group between the cooked glutinous and non-glutinous sorghum samples, the intensities of each group were summed (Table 4). 
Table 3. Aroma compounds in cooked glutinous and non-glutinous sorghum.

\begin{tabular}{|c|c|c|c|c|c|c|c|}
\hline \multirow{2}{*}{ No. ${ }^{a}$} & \multirow{2}{*}{ Compound } & \multirow{2}{*}{ Aroma Descriptor } & \multicolumn{2}{|c|}{ Aroma Intensity ${ }^{b}$} & \multirow{2}{*}{ Significant $^{c}$} & \multirow{2}{*}{ LRI $^{d}$} & \multirow{2}{*}{ Identification } \\
\hline & & & Glutinous Sorghum & Non-Glutinous Sorghum & & & \\
\hline \multicolumn{8}{|c|}{ Aldehydes } \\
\hline 1 & 2-Methylbutanal & Green, malty & $3.25 \pm 0.27$ & $3.08 \pm 0.20$ & ns & 912 & MS, RI, Aroma, STD \\
\hline 2 & 3-Methylbutanal & Green, malty & $4.08 \pm 0.49$ & $4.17 \pm 0.25$ & ns & 925 & MS, RI, Aroma, STD \\
\hline 4 & Hexanal & Grassy, green & $2.92 \pm 0.38$ & $2.58 \pm 0.37$ & ns & 1084 & MS, RI, Aroma, STD \\
\hline 11 & Octanal & Fatty, soapy, orange & $2.83 \pm 0.41$ & $3.17 \pm 0.25$ & ns & 1289 & MS, RI, Aroma, STD \\
\hline 13 & (E)-2-Heptenal & Fatty & $2.83 \pm 0.61$ & $2.00 \pm 0.45$ & $*$ & 1315 & MS, RI, Aroma, STD \\
\hline 15 & Nonanal & Green & $4.33 \pm 0.52$ & $2.50 \pm 0.20$ & $* * *$ & 1396 & MS, RI, Aroma, STD \\
\hline 17 & (E)-2-octenal & Green & $4.58 \pm 0.38$ & $4.00 \pm 0.45$ & $*$ & 1435 & MS, RI, Aroma, STD \\
\hline 22 & Decanal & Fatty/waxy & $2.50 \pm 0.45$ & $2.08 \pm 0.51$ & ns & 1506 & MS, RI, Aroma, STD \\
\hline 23 & Benzaldehyde & Almond & $5.00 \pm 0.00$ & $4.33 \pm 0.25$ & $* *$ & 1541 & MS, RI, Aroma, STD \\
\hline 30 & (E)-2-decenal & Green & $1.00 \pm 0.71$ & $0.83 \pm 0.45$ & ns & 1656 & MS, RI, Aroma, STD \\
\hline 31 & Phenylacetaldehyde & Rose & $1.50 \pm 0.45$ & $2.00 \pm 0.38$ & ns & 1675 & MS, RI, Aroma, STD \\
\hline 33 & $(E, E)-2,4$-Nonadienal & Green, fatty & $2.83 \pm 0.61$ & $3.08 \pm 0.45$ & ns & 1711 & MS, RI, Aroma, STD \\
\hline 37 & (E)-2-Undecenal & Soapy, metallic & - & $0.92 \pm 0.32$ & $* * *$ & 1736 & MS, RI, Aroma, STD \\
\hline 38 & $(E, E)-2,4$-Decadienal & Fatty, vegetable & $1.75 \pm 0.27$ & - & $* * *$ & 1820 & MS, RI, Aroma, STD \\
\hline \multicolumn{8}{|c|}{ Terpenes } \\
\hline 7 & $\alpha$-Limonene & Citrus-like & $1.25 \pm 0.69$ & - & $* *$ & 1270 & MS, RI, Aroma, STD \\
\hline 9 & $\gamma$-Terpinene & Citrus-like, lemon & $2.25 \pm 0.52$ & $2.00 \pm 0.41$ & ns & 1273 & MS, RI, Aroma, STD \\
\hline 24 & $(-)-\alpha$-Cedrene & Cypress & $5.00 \pm 0.00$ & $3.17 \pm 0.40$ & $* * *$ & 1574 & MS, RI, Aroma, STD \\
\hline 25 & Thujopsene & Cypress & $1.50 \pm 0.45$ & - & $* * *$ & 1620 & MS, RI, Aroma, STD \\
\hline 28 & $\beta$-Terpineol & Clove & $0.92 \pm 0.49$ & $1.33 \pm 0.38$ & ns & 1626 & MS, RI, Aroma, STD \\
\hline 36 & $\beta$-Elemene & Fruity, sweet & $1.83 \pm 0.41$ & - & $* * *$ & 1598 & MS, RI, Aroma, STD \\
\hline 39 & $\beta$-Damascenone & Sweet, honey & $5.00 \pm 0.00$ & $4.08 \pm 0.45$ & $* *$ & 1833 & MS, RI, Aroma, STD \\
\hline 42 & $(E)-\beta$-Ionone & Violet & $2.25 \pm 0.52$ & $1.75 \pm 0.38$ & ns & 1952 & MS, RI, Aroma, STD \\
\hline 45 & Geranyl acetone & Fruity & $5.00 \pm 0.00$ & $4.25 \pm 0.25$ & $* * *$ & 1856 & MS, RI, Aroma, STD \\
\hline 46 & (E)-Nerolidol & Rose, apple & $1.50 \pm 0.45$ & $1.92 \pm 0.37$ & ns & 2015 & MS, RI, Aroma, STD \\
\hline 52 & Vanillin & Sweet & $1.83 \pm 0.61$ & $2.17 \pm 0.51$ & ns & 2542 & MS, RI, Aroma, STD \\
\hline
\end{tabular}


Table 3. Cont.

\begin{tabular}{|c|c|c|c|c|c|c|c|}
\hline \multirow{2}{*}{ No. ${ }^{a}$} & \multirow{2}{*}{ Compound } & \multirow{2}{*}{ Aroma Descriptor } & \multicolumn{2}{|c|}{ Aroma Intensity ${ }^{b}$} & \multirow{2}{*}{ Significant ${ }^{c}$} & \multirow{2}{*}{ LRI $^{d}$} & \multirow{2}{*}{ Identification } \\
\hline & & & Glutinous Sorghum & Non-Glutinous Sorghum & & & \\
\hline \multicolumn{8}{|l|}{ Acids } \\
\hline 20 & Acetic acid & Acid & $0.83 \pm 0.52$ & $1.50 \pm 0.49$ & ns & 1455 & MS, RI, Aroma, STD \\
\hline 29 & Butanoic acid & Sweaty, acid & $2.17 \pm 0.41$ & $2.67 \pm 0.66$ & ns & 1657 & MS, RI, Aroma, STD \\
\hline 34 & 3-Methyl-butanoic acid & Acid & $2.25 \pm 0.52$ & $2.50 \pm 0.55$ & ns & 1687 & MS, RI, Aroma, STD \\
\hline 35 & Pentanoic acid & Sweaty & $2.25 \pm 0.27$ & $2.58 \pm 0.45$ & ns & 1754 & MS, RI, Aroma, STD \\
\hline 40 & Hexanoic acid & Sweaty, cheese & $5.00 \pm 0.00$ & $5.00 \pm 0.00$ & ns & 1844 & MS, RI, Aroma, STD \\
\hline 43 & Heptanoic acid & Sweaty & $1.67 \pm 0.26$ & $2.17 \pm 0.68$ & ns & 1915 & MS, RI, Aroma, STD \\
\hline 49 & Octanoic acid & Grease & $1.75 \pm 0.61$ & $2.08 \pm 0.68$ & ns & 2060 & MS, RI, Aroma, STD \\
\hline 51 & Decanoic acid & Grease & $3.17 \pm 0.68$ & $3.50 \pm 0.45$ & ns & 2284 & MS, RI, Aroma, STD \\
\hline \multicolumn{8}{|c|}{ Ketones } \\
\hline 10 & 2-Octanone & Milk & $1.58 \pm 0.49$ & $1.83 \pm 0.58$ & ns & 1282 & MS, RI, Aroma, STD \\
\hline 12 & 2,3-Octanedione & Cream, sweet & $3.33 \pm 0.41$ & $2.67 \pm 0.20$ & $* *$ & 1368 & MS, RI, Aroma, STD \\
\hline 14 & 2-Nonanone & Sweet, coconut & $1.17 \pm 0.41$ & $1.00 \pm 0.45$ & ns & 1388 & MS, RI, Aroma, STD \\
\hline 27 & 2-Undecanone & Orange & $1.17 \pm 0.61$ & $1.58 \pm 0.45$ & ns & 1596 & MS, RI, Aroma, STD \\
\hline 32 & Acetophenone & Floral & $4.00 \pm 0.45$ & $3.08 \pm 0.51$ & $*$ & 1668 & MS, RI, Aroma, STD \\
\hline \multicolumn{8}{|c|}{ Alcohols } \\
\hline 16 & Hexanol & Floral & $3.50 \pm 0.71$ & $3.00 \pm 0.38$ & ns & 1376 & MS, RI, Aroma, STD \\
\hline 19 & Heptanol & Green & $0.92 \pm 0.49$ & $1.17 \pm 0.38$ & ns & 1450 & MS, RI, Aroma, STD \\
\hline 26 & Octanol & Fruity & $2.50 \pm 0.63$ & $2.75 \pm 0.51$ & ns & 1575 & MS, RI, Aroma, STD \\
\hline 41 & Phenethyl alcohol & Honey & $3.50 \pm 0.45$ & $4.17 \pm 0.60$ & ns & 1942 & MS, RI, Aroma, STD \\
\hline \multicolumn{8}{|c|}{ Heterocyclics } \\
\hline 8 & 2-Pentylfuran & Grass, bean & $2.92 \pm 0.58$ & $2.00 \pm 0.38$ & $*$ & 1218 & MS, RI, Aroma, STD \\
\hline 21 & Furfural & Almond & $1.17 \pm 0.61$ & $1.00 \pm 0.58$ & ns & 1464 & MS, RI, Aroma, STD \\
\hline 44 & 2-Acetylpyrrole & Popcorn-like & $0.75 \pm 0.42$ & $1.83 \pm 0.45$ & $* *$ & 1957 & MS, RI, Aroma, STD \\
\hline 47 & $\gamma$-Octalactone & Coconut milk & $3.50 \pm 0.63$ & $3.00 \pm 0.74$ & ns & 1886 & MS, RI, Aroma, STD \\
\hline 48 & $\gamma$-Nonalactone & Coconut milk & $2.92 \pm 0.58$ & $2.50 \pm 0.38$ & ns & 2059 & MS, RI, Aroma, STD \\
\hline \multicolumn{8}{|c|}{ Sulphur } \\
\hline $\begin{array}{c}3 \\
\text { Aromat }\end{array}$ & Dimethyl disulfide & Onion & $2.00 \pm 0.32$ & $1.17 \pm 0.38$ & $* *$ & 1078 & MS, RI, Aroma, STD \\
\hline 50 & $\begin{array}{l}\text { 2-Methoxy-4- } \\
\text { vinylphenol }\end{array}$ & Smoky, clove-like & $2.25 \pm 0.52$ & $2.50 \pm 0.63$ & ns & 2200 & MS, RI, Aroma, STD \\
\hline
\end{tabular}

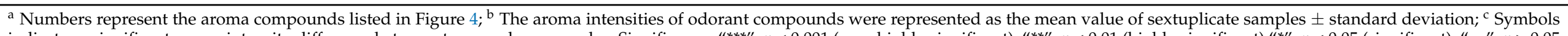

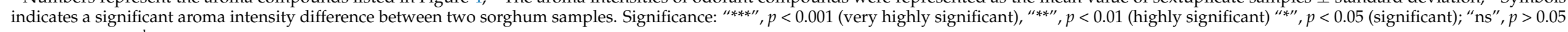
(not significant). ${ }^{\mathrm{d}}$ LRI: linear retention indices. 


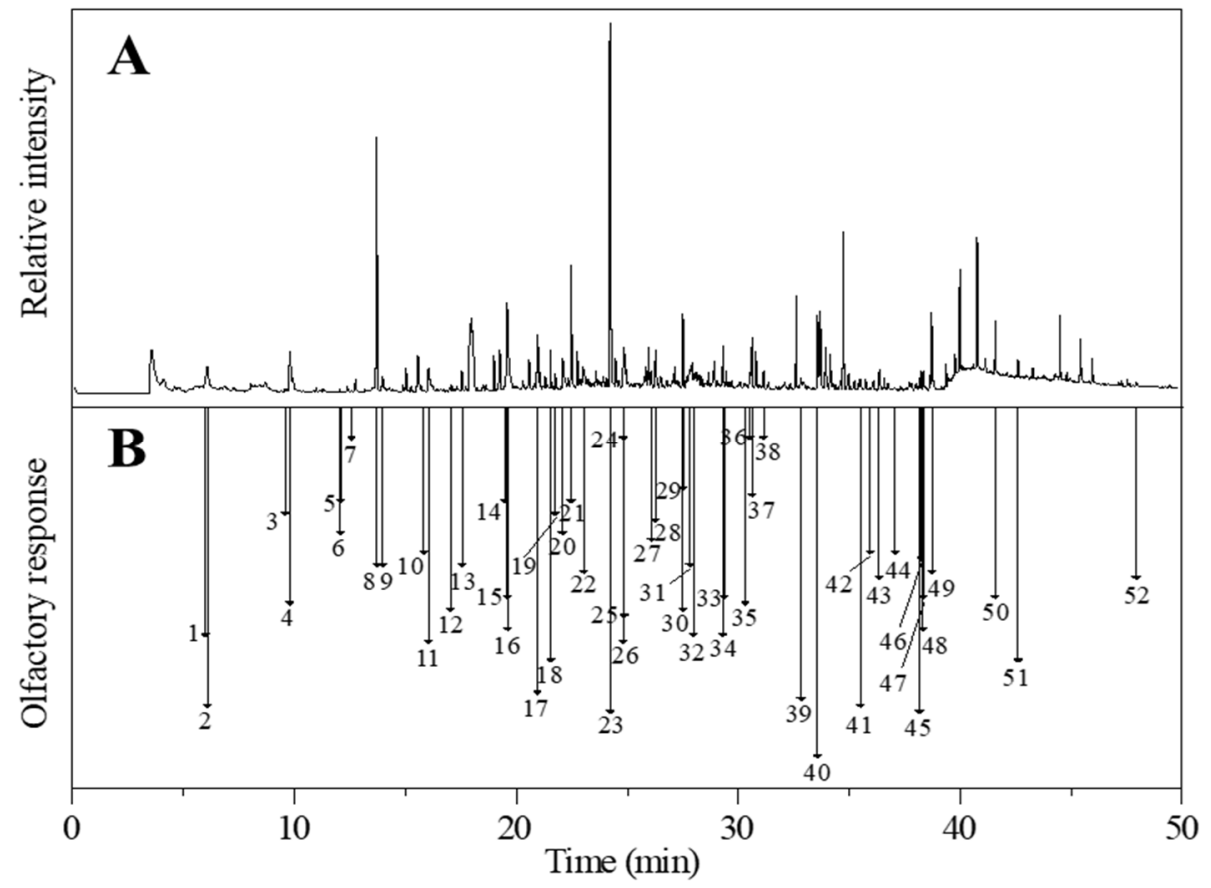

Figure 4. (A) Gas chromatography-mass spectrometry (GC-MS) and (B) gas chromatographyolfactory (GC-O) chromatograms of cooked glutinous sorghum. Numbers correspond to the compounds listed in Table 3.

Table 4. Summed aroma intensities of each aroma group present in the cooked glutinous and non-glutinous sorghum samples.

\begin{tabular}{|c|c|c|c|c|}
\hline \multirow{2}{*}{ Aroma Impression ${ }^{a}$} & \multirow{2}{*}{ Volatile Compounds } & \multicolumn{2}{|c|}{ Summed Aroma Intensities ${ }^{b}$} & \multirow{2}{*}{ Significance } \\
\hline & & Glutinous & Non-Glutinous & \\
\hline sweet/fruity & $\begin{array}{c}\text { 2-octanone, } 2,3 \text {-octanedione, } 2 \text {-nonanone, octanol, } \beta \text {-elemene, } \\
\beta \text {-damascenone, phenethyl alcohol, geranyl acetone, } \\
\gamma \text {-octalactone, } \gamma \text {-nonalactone, vanillin }\end{array}$ & $32.17 \pm 2.32$ & $28.42 \pm 1.48$ & $*$ \\
\hline green & $\begin{array}{l}\text { 2-methylbutanal, 3-methylbutanal, hexanal, 2-pentylfuran, } \\
\text { nonanal, }(E) \text {-2-octenal, 1-heptanol, (-)- } \alpha \text {-cedrene, thujopsene, } \\
(E) \text {-2-decenal, }(E, E) \text {-2,4-nonadienal }\end{array}$ & $33.33 \pm 1.75$ & $26.58 \pm 1.51$ & $* * *$ \\
\hline floral & $\begin{array}{l}\text { hexanol, } \beta \text {-terpineol, phenylacetaldehyde, acetophenone, } \\
\text { (E)- } \beta \text {-ionone, (E)-nerolidol }\end{array}$ & $13.67 \pm 1.67$ & $13.08 \pm 1.51$ & $\mathrm{~ns}$ \\
\hline fatty & $\begin{array}{l}\text { octanal, }(E) \text {-2-heptenal, decanal, }(E) \text {-2-undecenal, }(E, \\
E) \text {-2,4-decadienal, octanoic acid, decanoic acid }\end{array}$ & $14.83 \pm 0.75$ & $13.75 \pm 1.80$ & ns \\
\hline volatile acids & $\begin{array}{l}\text { acetic acid, butanoic acid, 3-methyl-butanoic acid, pentanoic } \\
\text { acid, hexanoic acid, heptanoic acid }\end{array}$ & $14.17 \pm 0.75$ & $16.42 \pm 1.06$ & $* *$ \\
\hline citrus-like & $\begin{array}{l}\alpha \text {-terpinene, }(E) \text { - } \beta \text {-terpinolene, } \alpha \text {-limonene, } \gamma \text {-terpinene, } \\
2 \text {-undecanone }\end{array}$ & $7.67 \pm 0.55$ & $6.08 \pm 0.53$ & $* * *$ \\
\hline roasty/nutty & furfural, benzaldehyde, 2-acetylpyrrole & $6.92 \pm 0.61$ & $7.17 \pm 0.69$ & ns \\
\hline $\begin{array}{l}\text { miscellaneous (sul- } \\
\text { phury/smoky/mushroom) }\end{array}$ & dimethyl disulfide, 1-octen-3-ol, 2-methoxy-4-vinylphenol & $7.58 \pm 0.79$ & $7.17 \pm 0.47$ & ns \\
\hline
\end{tabular}

${ }^{a}$ The aroma characteristics; ${ }^{b}$ The summed aroma intensities of odorant compounds were represented as the mean value of sextuplicate samples \pm standard deviation; ${ }^{c}$ Symbols indicate a significant aroma intensity difference between two sorghum samples. Significance: “***”, $p<0.001$ (very highly significant); “**”, $p<0.01$ (highly significant) “*”, $p<0.05$ (significant); "ns", $p>0.05$ (not significant).

\subsubsection{Sweet/Fruity}

Sweet/fruity was found to be the major aroma character in both types of cooked sorghums, with the glutinous sorghum containing $12 \%$ more sweet/fruity aroma than the non-glutinous sorghum. Four types of compounds (terpenes, heterocyclics, alcohols, and ketones) provided the sweet/fruity aroma note in the cooked sorghums. Among the four terpenes (elemene, $\beta$-damascenone, geranyl acetone, and vanillin), $\beta$-damascenone and geranyl acetone were significant contributors to the overall aroma of the cooked 
sorghums. During the GC-O/MS analysis, although no obvious peak for $\beta$-damascenone was observed, an aroma of rich honey sweetness was sniffed at $26.84 \mathrm{~min}$, with an RI of 1833 (Figure 4), and the compound was identified using its retention time and mass ions. $\beta$-Damascenone is an ionone isomer belonging to the isoprenoid class of compounds. These compounds often exude the aroma of lemon at low concentrations, while at high concentrations the aromas of apple, rose, and honey are expressed [16]. According to the literature, $\beta$-damascenone is an important aroma contributor to Chinese Baijiu [6], having extremely low aroma threshold values in air $(0.002-0.004 \mathrm{ng} / \mathrm{L})$ and water $(0.00075$ $0.002 \mu \mathrm{g} / \mathrm{L}$ ) [17]. $\beta$-Damascenone and geranyl acetone are both bound aroma compounds of sorghum, with geranyl acetone also being an important aroma compound in the soy sauce aroma type Baijiu [6]. Vanillin is another common aroma compound in Chinese Baijiu [18]. It has a sweet vanilla aroma feature with a low aroma threshold of $26 \mu \mathrm{g} / \mathrm{L}$ in chinese rice wine $[17,19]$. The two sweet/fruity heterocyclic compounds $(\gamma$-octalactone and $\gamma$-nonalactone) contributed a coconut aroma to the cooked sorghums. Both compounds are $\gamma$-lactones, a significant class of aroma lactones that mostly have coconut, sweet, and peach aromas [20]. $\gamma$-Octalactone and $\gamma$-nonalactone also have relatively low aroma thresholds, 7 and 30-65 $\mu \mathrm{g} / \mathrm{L}$, respectively. Additionally, two alcohols and two ketones contributed to the sweet/fruity aromas of the cooked sorghums. Among these, phenethyl alcohol was a significant aroma contributor having an aroma intensity of 3.50 and 4.17 in glutinous and non-glutinous sorghum, respectively (Table 3 ).

\subsubsection{Green}

Green was another significant aroma note in the cooked sorghums. In this study, 11 green aroma compounds were detected, having total aroma intensities $20 \%$ higher in glutinous versus non-glutinous sorghum (Table 4). Seven of the eleven compounds were aldehydes. Saturated aldehydes and (E)-2-enal unsaturated aldehydes often demonstrate grass and malty aromas [14,17]. Nonanal and 3-methylbutanal (saturated aldehydes) and (E)-2-octenal (an unsaturated aldehyde), were evaluated as having strong aroma intensities in both cooked sorghums. Lipids in sorghum are susceptible to oxidization, and the heat imposed during sorghum distillation may accelerate the oxidation process [21], possibly contributing to the presence of aldehyde compounds in the cooked sorghum samples. (E)-2-Enal aldehydes have not been detected in uncooked sorghum [10,11], while they have been detected in a variety of Chinese Baijiu [14], thus further substantiating that the distillation (cooking) process of sorghum may improve the release of bound aroma compounds and these compounds influence the final flavor quality of Baijiu. (-)- $\alpha$-Cedrene, a terpene, showed the strongest green aroma note (5.0) in glutinous sorghum (Table 3). As (-)- $\alpha$-Cedrene is also an aroma compound in the soy sauce aroma type Baijiu, this compound may strongly contribute to the overall aroma of Baijiu [22].

\subsubsection{Floral}

Six compounds were detected as having floral aromas in the cooked sorghums. The summed aroma intensities of these compounds for the two types of sorghum were similar (Table 4). Of the compounds primarily responsible for the floral aromas of the cooked sorghums, acetophenone had the highest relative floral aroma intensity, 4.00 and 3.08 in glutinous and non-glutinous sorghum, respectively.

\subsubsection{Fatty}

Seven compounds were detected as having fatty aromas. Compounds with long carbon chains, such as octanal and decanoic acid, often demonstrate fatty aromas. However, with decreases in the carbon chain length, compounds may demonstrate different aroma notes, and their aroma thresholds may also decrease [17]. 


\subsubsection{Volatile Acids}

Organic acids are important aroma compounds in many traditional fermented foods [23]. Six compounds having acids aromas were detected, and their summed aroma intensities were $14 \%$ lower in glutinous versus non-glutinous sorghum (Table 4). Among these, hexanoic acid was evaluated as having sweaty and cheese aromas, with aroma intensities of 5.00 for both types of cooked sorghums (Table 3).

\subsubsection{Citrus-Like}

The citrus-like aroma is a unique feature of the aroma profile of cooked sorghums. Four terpenes and one ketone were detected with citrus-like aromas. Among these, $\gamma$-terpinene showed a relatively strong aroma intensity of 2.00 both in glutinous and non-glutinous sorghum (Table 3).

\subsubsection{Roasty/Nutty}

Three compounds were detected as having roasty/nutty aromas. Benzaldehyde demonstrated strong almond aroma intensities of 5.00 and 4.33 in glutinous and nonglutinous sorghum, respectively (Table 3).

\subsubsection{Miscellaneous}

Besides the typical aroma notes of cooked sorghum, 1-octen-3-ol (mushroom aroma), dimethyl disulfide (onion aroma), and 2-methoxy-4-vinylphenol (smoky aroma), were also detected and contributed to the overall aromas of the cooked sorghums (Table 3).

Based on the ANOVA, the summed intensity of four aroma categories: sweet/fruity, green, volatile acids and citrus-like, present significant differences between glutinous and non-glutinous sorghum samples (Table 4).

\section{Materials and Methods}

\subsection{Reagents and Chemicals}

Two sorghum cultivars (glutinous and non-glutinous) produced in Guizhou Province in 2018, were provided by a liquor producing enterprise. The aroma standards were purchased from Sigma Aldrich (Shanghai) Trade Co. Ltd. All compounds were of chromatographic purity, and the purity was $>95 \%$. The n-alkane (C7-C30) for the linear retention index (RI) determination was obtained from Sigma-Aldrich Co., Ltd. (Shanghai, China). Ultrapure water was obtained from a Milli-Q purification system (Millipore, Bedford, MA, USA).

\subsection{Sorghum Cooking}

Sorghum samples were first moistened by adding $300 \mathrm{~g}$ of sorghum to $400 \mathrm{~mL}$ of distilled water in a $1 \mathrm{~L}$ beaker. The mixture was then sealed with foil and heated in an oven at $75{ }^{\circ} \mathrm{C}$ overnight [24]. Then, $5 \mathrm{~g}$ of the moist sorghum and $1 \mathrm{~mL}$ of distilled water were added to a $20 \mathrm{~mL}$ headspace glass vial that was purged with nitrogen gas and loosely sealed with a Teflon-coated septum screw cap. Next, the vial was placed in a boiling water bath $\left(100^{\circ} \mathrm{C}\right)$ for $1 \mathrm{~h}$, and directly subjected to headspace-solid-phase microextraction.

\subsection{Optimization of Headspace-Solid-Phase Microextraction Sampling}

Four SPME fiber types, polydimethylsiloxane (PDMS), PDMS/divinylbenzene (DVB), carboxen (CAR)/PDMS, and DVB/CAR/PDMS, were evaluated for their capabilities at extracting and concentrating the headspace volatiles of cooked sorghum. Extraction temperatures $\left(40,50,60,70\right.$, and $\left.80^{\circ} \mathrm{C}\right)$ and times $(30,60,90,120$, and $150 \mathrm{~min}$ ) were evaluated to determine the optimal conditions for extracting the volatiles using each type of SPME fiber. After optimization, the DVB/CAR/PDMS SPME fiber was inserted into the headspace vial of the sorghum sample and left for $120 \mathrm{~min}$ at $80^{\circ} \mathrm{C}$. Subsequently, the fiber was inserted into the injection port of the GC-MS for $5 \mathrm{~min}$ at $250^{\circ} \mathrm{C}$ for thermal desorption of the flavor volatiles. 


\subsection{HS-SPME-GC $\times$ GC-TOFMS Instrumentation}

Following a method presented in the literature [25], experiments were performed on a LECO Pegasus ${ }^{\circledR}$ 4D GC $\times$ GC-TOFMS system (LECO Corp., St. Joseph, MI, USA). The column configurations for GC $\times \mathrm{GC}$ analysis consisted of two columns which were coupled in tandem. The 1D column was a $60 \mathrm{~m} \times 0.25 \mathrm{~mm} \times 0.25 \mu \mathrm{m}$ DB-FFAP (Agilent Technologies, Palo Alto, CA, USA), and the 2D column was a $1.5 \mathrm{~m} \times 0.25 \mathrm{~mm} \times 0.25 \mu \mathrm{m}$ Rxi-17Sil MS secondary column (Restek, Bellefonte, PA, USA). Helium was used as the carrier gas at a constant flow rate of $1.0 \mathrm{~mL} / \mathrm{min}$. The injector temperature was set at $250{ }^{\circ} \mathrm{C}$. The initial oven temperature was held at $45^{\circ} \mathrm{C}$ for $3 \mathrm{~min}$, and then ramped at a rate of $4{ }^{\circ} \mathrm{C} / \mathrm{min}$ to $150{ }^{\circ} \mathrm{C}$, and held for $2 \mathrm{~min}$, reaching $200{ }^{\circ} \mathrm{C}$ at $6{ }^{\circ} \mathrm{C} / \mathrm{min}$ and $230{ }^{\circ} \mathrm{C}$ at $10{ }^{\circ} \mathrm{C} / \mathrm{min}$ for $20 \mathrm{~min}$. The secondary oven was set at a $5^{\circ} \mathrm{C}$ offset, relative to the primary oven. The modulator was set at a $20^{\circ} \mathrm{C}$ offset, relative to the primary oven, and a modulation period of $4 \mathrm{~s}$ was used. The TOFMS was operated at an acquisition rate of 100 spectra/s and scanned from $50 \mathrm{~m} / \mathrm{z}$ to $350 \mathrm{~m} / \mathrm{z}$.

The detector voltage was set to $1350 \mathrm{~V}$, and the electron energy was $-70 \mathrm{~V}$.

\subsection{Gas Chromatography-Olfactometry/Mass Spectrometry (GC-O/MS) Analysis}

Gas chromatography was performed using an Agilent 6890N GC equipped with an Agilent 5975 mass selective detector (Agilent Technologies, Santa Clara, CA) and olfactometer (ODP 2, Gerstel GmbH and Co. KG, Mulheim, Germany). Samples were separated and evaluated using a polar DB-FFAP column $(60 \mathrm{~m} \times 0.25 \mathrm{~mm}$ i.d, $0.25 \mu \mathrm{m}$ film thickness, Agilent Technologies, Inc., Santa Clara, CA). The oven temperature was programmed to $40{ }^{\circ} \mathrm{C}$ for $2 \mathrm{~min}$, then increased at a rate of $4{ }^{\circ} \mathrm{C} / \mathrm{min}$ to $230{ }^{\circ} \mathrm{C}$, and maintained there for $10 \mathrm{~min}$. The carrier gas used was helium, at a flow rate of $2.0 \mathrm{~mL} / \mathrm{min}$. A split ratio of 1:1 was used for injection.

Half of the carrier flow was diverted to the olfactory port, and mixed with warm, humid air for sniffing. The sniffing port temperature was set to $250{ }^{\circ} \mathrm{C}$. Three trained panelists (two females and one male) received two months of training on GC-O involving, at a minimum, 30 aroma-active reference compounds at concentrations that were 10 times their odor thresholds in air. During GC operation, each panelist placed his/her nose near the olfactory port to record the retention times, intensity values, and aroma descriptions of the samples. Odor intensities were evaluated using a 6-point intensity scale from 0 to 5 , with " 0 " = no, " 3 " = moderate, and " 5 " = extreme intensity. Each panelist conducted the GC-O evaluation four times. The final intensity value of each aroma was an average of the aroma intensities obtained from all of the panelists.

The other half of the carrier flow was diverted to the mass spectrometer. The ion source temperature was set to $230{ }^{\circ} \mathrm{C}$, electron impact ionization was applied in positive ion mode $(70 \mathrm{eV})$, and the mass range scanned was $\mathrm{m} / \mathrm{z}=25 \sim 300$.

\subsection{Identification of Aroma Compounds}

Identifications were made through comparisons of the aromas, mass spectra, and linear retention indices (LRIs) with reference/standard compounds. Aroma determinations were made by comparing the odor descriptions of the compounds analyzed by GC-O to Flavornet and Flavor DB (citations). Mass determinations were made by comparing the mass spectra of the compounds to the NIST/EPA/NIH mass spectral library (NIST 14, version 2.0) (NIST, Gaithersburg, MD). LRI analyses involved comparisons of the aroma LRIs with those of reference compounds. The LRIs of the odorants were calculated from the linear retention times of n-alkanes (C5-C30) using a DB-FFAP column according to a modified Kovats method [26]. All of the aroma-active compounds detected by GC-O were also confirmed through the use of standard compounds. 


\subsection{Statistical Analysis}

The analysis of variance (ANOVA) was performed using Microsoft Excel 2013 software. Significant differences were estimated as “***”, $p<0.001$ (very highly significant); "“**”, $p<0.01$ (highly significant); “*", $p<0.05$ (significant); "ns", $p>0.05$ (not significant).

\section{Conclusions}

In this study, an SPME method for the extraction of aroma compounds from cooked sorghums was developed. Using this method, a total of 145 volatile compounds and 52 aroma-active compounds were detected in cooked glutinous and non-glutinous sorghum by GC $\times$ GC-TOFMS and GC-O/MS respectively. These compounds were further identified through comparisons with reference/standard compounds. The aroma features and intensities of these compounds were evaluated by three well-trained panelists using olfactory detection. The aroma intensity of $(E)$-2-heptenal, nonanal, $(E)$-2-octenal, benzaldehyde, (E)-2-undecenal, (E, E)-2,4-decadienal, $\alpha$-limonene, (-)- $\alpha$-Cedrene, thujopsene, $\beta$ elemene, $\beta$-damascenone, geranyl acetone, 2,3-octanedione, acetophenone, 2-pentylfuran, 2-acetylpyrrole and dimethyl disulfide, present significant differences between the two samples $(p<0.05)$. $(E)$-2-undecenal was undetected in glutinous sorghum, and $(E, E)-2,4-$ decadienal, $\alpha$-limonene, thujopsene, and $\beta$-elemene were undetected in non-glutinous sorghum. Based on their aroma features, the compounds were grouped into eight general aroma categories, and the aroma intensities of each aroma group were totaled. The summed intensity of four aroma categories: sweet/fruity, green, volatile acids and citruslike, present significant differences between glutinous and non-glutinous sorghum samples $(p<0.05)$. Glutinous sorghum contained $12 \%, 20 \%$ and $21 \%$ more sweet/fruity, green and citrus-like total intensities, respectively than non-glutinous sorghum; and contained $14 \%$ less acid total intensity, emphasizing the aromatic differences between the two cooked sorghums. In addition, most of the compounds detected in the cooked sorghums were also detected in commercial Baijiu, indicating that the aroma compounds produced during sorghum distillation (cooking) have direct and significant influences on the final flavor quality of Baijiu.

Author Contributions: S.C., Conceptualization, Methodology, Validation, Analysis, Writing, Review \& Editing, project administration; L.W., Supervision \& Review; D.N., Validation, Analysis; L.L., Methodology, Analysis; H.W., Methodology, Validation, Analysis; Y.X., Supervision \& Review. All authors have read and agreed to the published version of the manuscript.

Funding: This research was funded by the National Key R\&D Program of China (NO. 2018YFC1604100), the China Postdoctoral Science Foundation (NO. 2018M631971), National First-class Discipline Program of Light Industry Technology and Engineering (LITE2018-12), and 111 Program of Introducing Talents (NO. 111-2-06).

Institutional Review Board Statement: Not applicable.

Informed Consent Statement: Not applicable.

Data Availability Statement: The data presented in this study are available on request from the corresponding author.

Conflicts of Interest: The authors declare no conflict of interest.

Sample Availability: Samples of the compounds are not available from the authors.

\section{References}

1. Mahama, G.Y.; Prasad, P.; Mengel, D.B.; Tesso, T.T. Influence of nitrogen fertilizer on growth and yield of grain sorghum hybrids and inbred lines. Agron. J. 2014, 106, 1623-1630. [CrossRef]

2. Shen, Y. Manual of Chinese Liquor Manufactures Technology; Light Industry Publishing House of China: Beijing, China, 1996.

3. Jin, G.; Zhu, Y.; Xu, Y. Mystery behind Chinese liquor fermentation. Trends Food Sci. Technol. 2017, 63, 18-28. [CrossRef]

4. Chen, B.; $\mathrm{Wu}, \mathrm{Q}$.; $\mathrm{Xu}, \mathrm{Y}$. Filamentous fungal diversity and community structure associated with the solid state fermentation of Chinese Maotai-flavor liquor. Int. J. Food Microbiol. 2014, 179, 80-84. [CrossRef] 
5. Fan, X.; Jiao, X.; Liu, J.; Jia, M.; Blanchard, C.; Zhou, Z. Characterizing the volatile compounds of different sorghum cultivars by both GC-MS and HS-GC-IMS. Food Res. Int. 2021, 140, 109975. [CrossRef] [PubMed]

6. Wang, X.; Fan, W.; Xu, Y. Comparison on aroma compounds in Chinese soy sauce and strong aroma type liquors by gas chromatography-olfactometry, chemical quantitative and odor activity values analysis. Eur. Food Res. Technol. 2014, 239, 813-825. [CrossRef]

7. Gao, W.; Fan, W.; Xu, Y. Characterization of the key odorants in light aroma type Chinese liquor by gas chromatographyolfactometry, quantitative measurements, aroma recombination, and omission studies. J. Agric. Food Chem. 2014, 62, 5796-5804. [CrossRef]

8. $\mathrm{Xu}, \mathrm{Y}$. Study on liquor-making microbes and the regulation \& control of their metabolism based on flavor-oriented technology. Liquor. Mak. Sci. Technol. 2015, 2, 1-11.

9. Liu, H.; Sun, B. Effect of Fermentation Processing on the Flavor of Baijiu. J. Agric. Food Chem. 2018, 66, 5425-5432. [CrossRef] [PubMed]

10. Lian, S.; Xie, Z.; Huaxia, Y.E.; Yanghua, L.I.; Wang, F.; Yunhong, W.U. Research on the Flavouring Compositions of Sorghum. Liquor Mak. Sci. Technol. 2012, 3, 40-43.

11. Wu, Y.; Liu, S.; Fan, X.; Yang, J.; Jiang, W.; Wang, D.; Li, N. Analysis of Aroma Components of Five Different Cooked Grains Used for Chinese Liquor Production by GC-O-MS. Food Sci. 2016, 37, 94-98.

12. Turiel, E.; Martín-Esteban, A. Molecularly imprinted polymers for solid-phase microextraction. J. Sep. Sci. 2009, 32, 3278-3284 [CrossRef]

13. Zeng, Z.; Zhang, H.; Chen, J.Y.; Zhang, T.; Matsunaga, R. Direct Extraction of Volatiles of Rice During Cooking Using Solid-Phase Microextraction. Cereal Chem. 2007, 84, 423-427. [CrossRef]

14. Cao, C.; Fan, W.; Nie, Y.; Xu, Y. Quantification of trans-2- alkenals and alkadienals in Chinese liquors by simultaneous extraction/derivatization by HS- SPME coupled with GC- MS- SIM. Sci. Technol. Food Ind. 2014, 21, 286-290.

15. Wang, L.; Fan, S.; Yan, Y.; Yang, L.; Chen, S.; Xu, Y. Characterization of Potent Odorants Causing a Pickle-like Off-Odor in Moutai-Aroma Type Baijiu by Comparative Aroma Extract Dilution Analysis, Quantitative Measurements, Aroma Addition, and Omission Studies. J. Agric. Food Chem. 2020, 68, 1666-1677. [CrossRef]

16. Pineau, B.; Barbe, J.-C.; Van Leeuwen, C.; Dubourdieu, D. Which impact for $\beta$-damascenone on red wines aroma? J. Agric. Food Chem. 2007, 55, 4103-4108. [CrossRef] [PubMed]

17. Fan, W.; Xu, Y. Flavor Chemistry of Alcoholic Beverage; China Light Industry Press: Beijing, China, 2014; pp. $117-121$.

18. Shi, D.; Wang, S.; Zhao, D.; Sun, J.; Li, A.; Sun, X.; Li, H.; Sun, B. Determination of 6 Phenols in 103 Kinds of Chinese Baijiu by GC-MS/SIM. J. Chin. Inst. Food Sci. Technol. 2019, 19, 235-248.

19. Chen, S.; Wang, C.; Qian, M.; Li, Z.; Xu, Y. Characterization of the Key Aroma Compounds in Aged Chinese Rice Wine by Comparative Aroma Extract Dilution Analysis, Quantitative Measurements, Aroma Recombination, and Omission Studies. J. Agric. Food Chem. 2019, 67, 4876-4884. [CrossRef]

20. Derail, C.; Hofmann, T.; Schieberle, P. Differences in key odorants of handmade juice of yellow-flesh peaches (Prunus persica L.) induced by the workup procedure. J. Agric. Food Chem. 1999, 47, 4742-4745. [CrossRef] [PubMed]

21. Zhao, T.; Chen, S.; Li, H.; Xu, Y. Determination of Linoleic Acid Oxylipins in Chinese Baijiu Using Ultra-Performance Liquid Chromatography with Quadruple-Time-of-Flight Mass Spectrometry (UPLC-QTOF-MS) and Nuclear Magnetic Resonance (NMR). Anal. Lett. 2019, 52, 2165-2179. [CrossRef]

22. Wang, L.; Hu, G.; Lei, L.; Lin, L.; Wang, D.; Wu, J. Identification and Aroma Impact of Volatile Terpenes in Moutai Liquor. Int. J. Food Prop. 2015, 19, 1335-1352. [CrossRef]

23. Belitz, H.; Grosch, W.; Schieberle, P. Food Chemistry; Springer: Berlin/Heidelberg, Germany, 1999.

24. Gao, L.; Tang, Q.; Tianxiang, W.U. Optimization of Fermentation-controlling Conditions of Bran-starter Maotai-flavor Liquor. Liquor Mak. Sci. Technol. 2011, 11, 40-43.

25. Wang, L.; Gao, M.; Liu, Z.; Chen, S.; Xu, Y. Three Extraction Methods in Combination with GCxGC-TOFMS for the Detailed Investigation of Volatiles in Chinese Herbaceous Aroma-Type Baijiu. Molecules 2020, 25, 4429. [CrossRef] [PubMed]

26. Cates, V.E.; Meloan, C.E. Separation of sulfones by gas chromatography. J. Chromatogr. A. 1963, 11, 472-478. [CrossRef] 